\title{
Water-Tank Studies of Separating Flow Over Rough Hills
}

\author{
J. B. R. Loureiro - A. S. Monteiro - F. T. Pinho • \\ A. P. Silva Freire
}

Received: 11 December 2007 / Accepted: 26 August 2008 / Published online: 30 September 2008

(C) Springer Science+Business Media B.V. 2008

\begin{abstract}
The present work investigates the lower boundary condition for flows over a steep, rough hill. Simple asymptotic arguments together with the mixing-length hypothesis are used to derive a local analytical solution that is tested against three different flow conditions. In all, 36 velocity profiles are compared with the proposed expression. The experiments were carried out in a water channel and velocity measurements were made through laser Doppler anemometry. The extent of separated flow was made to vary as a function of the roughness and the Reynolds number. The analysis includes regions of attached as well as separated flow. In particular, the solution of Stratford is studied at the points of separation and re-attachment and found to apply equally well in rough walls.
\end{abstract}

Keywords Hill $\cdot$ Law of the wall $\cdot$ Roughness $\cdot$ Separation

\section{Introduction}

Turbulent flows over steep hills have always presented considerable difficulties for their analytical treatment. The presence of large regions of reverse flow very often means that many of the classical theories and procedures are rendered non applicable. In particular, methods

J. B. R. Loureiro $(\varangle)$ · A. S. Monteiro · A. P. S. Freire

Programa de Engenharia Mecânica (COPPE/UFRJ), Universidade Federal do Rio de Janeiro, C.P. 68503, 21945-970 Rio de Janeiro, Brazil

e-mail: jbrloureiro@gmail.com

J. B. R. Loureiro

Divisão de Metrologia Científica e Industrial, Instituto Nacional de Metrologia (INMETRO), 22050-050 Rio de Janeiro, Brazil

F. T. Pinho

Centro de Estudos de Fenómenos de Transporte, Faculdade de Engenharia da Universidade do Porto,

Rua Dr. Roberto Frias s/n, 4200-465 Porto, Portugal

F. T. Pinho

Universidade do Minho, Largo do Paço, 4704-553 Braga, Portugal 
that rely on asymptotic solutions developed in terms of local scaling parameters based on the wall shear stress fail completely in the vicinity of a separation point.

In problems where hill perturbation effects are small enough, asymptotic expansion methods can be used to derive linearized equations of motion (Jackson and Hunt 1975; Sykes 1980; Hunt et al. 1998). By dividing the flow into distinct characteristic regions, the relevant dynamical processes that govern the problem can be modelled so that analytical expressions for the local mean velocity, pressure and Reynolds stress profiles can be obtained. For arbitrary hill shapes, these expressions can be quite evolving. Hence, a common practice has been to offer analytical solutions for hills that follow a Witch of Agnesi profile.

When the perturbation effects of the hill are strong enough to provoke a region of separated flow on its lee side, the fundamental hypotheses of linearized theory break down. The implication is that numerical models that consider the time averaged equations of motion have to be used. Indeed, many studies have been carried out over the last 20 years with the express purpose of analyzing the performance of several turbulence closures to model flow over steep hills. The recent and impressive advances in computing power have meant that simulations including first-order mixing-length schemes through second-order closure schemes have become commonplace (Castro and Apsley 1997; Ying and Canuto 1997; Hewer 1998; Ross et al. 2004). Even studies using much more demanding techniques such as large-eddy simulations have become quite frequent (Brown et al. 2001; Allen and Brown 2002; Iizuka and Kondo 2004).

An issue that has thus increased in importance is that of obtaining high-order closures that are capable of accurately modelling flows with separation and reattachment over hills. Procedures that specify the lower boundary condition based on logarithmic profiles need also to be reviewed since the physical arguments that imply their existence are violated near a separation point. The specification of the lower boundary condition is a particularly sensitive subject regarding the numerical prediction of flow properties. Kim and Patel (2000) have remarked that accurate predictions of a reverse flow region require the use of a low-Reynolds number model that can resolve the flow in the viscous sublayer. However, in atmospheric flows the Reynolds number is high and the presence of a rough wall means that there is no viscous sublayer. The implication is that the use of wall-functions becomes mandatory.

For flow over a smooth surface, Loureiro et al. (2007a) investigated the behaviour of four different near-wall formulations for separating flows: the classical logarithmic expression, and the expressions of Mellor (1966), Nakayama and Koyama (1984) and Cruz and Silva Freire $(1998,2002)$. The last three formulations have been specially advanced to deal with adverse pressure gradient flows: they reduce to the relevant wall laws at the appropriate limiting cases. Under attached flow conditions, they reduce to the classical logarithmic law of Prandtl (1925). Thus, $u_{*}\left(=\sqrt{\tau_{w} / \rho}\right)$ becomes the appropriate scaling velocity. Approaching a separation point, the proposed expressions reduce to Stratford's square-root law so that $u_{p v}\left(=\left((v / \rho) \partial_{x} p\right)^{1 / 3}\right)$ is the relevant scaling velocity.

The purpose of the present work is to investigate the lower boundary condition for separating flows over a rough hill. In particular, we concentrate on a parametric study of the near-wall formulation of Cruz and Silva Freire $(1998,2002)$ - previously derived for flow over a smooth surface - as it is applied to flow over a rough surface. Two new datasets from experiments conducted in a water channel are presented to characterize two-dimensional separated flow over a rough hill. For the first dataset, flow conditions and hill shape are the same as those of Loureiro et al. (2007a,b). However, the extent of separated flow is observed to be very much reduced due to the effects of the roughness. For the second dataset, the oncoming flow velocity is increased 10 fold. The magnitude of the separation region is then 
observed to increase significantly, but is still smaller than that reported by Loureiro et al. (2007a,b).

The experimental characterization of separating flow over rough surfaces is a subject that has long been recognized as deficient. The two reference datasets presented here are expected to provide an important complement to the data of Loureiro et al. (2007a,b), helping to establish rigorous conditions to which analytical solutions and numerical simulations of the problem can be tested. In addition, an important modification on the treatment of the lower boundary condition is introduced here. A new parametrization scheme is introduced to the additive parameter in the law of the wall so that the classical logarithmic and squaredrooted laws are recovered far away and near to a separation point. The new parametrization is defined in terms of $u_{*}, \partial_{x} p$ and $z_{0}$.

\section{Scaling Laws}

This section briefly reviews some basic arguments regarding the specification of the lower boundary condition for separating flow as well as for flow over a rough surface. Before tackling the rough surface problem, a short review on the smooth wall problem is presented. The purpose in doing this is to make the reader familiar with the flow structures near and far away from a separation point.

Asymptotic expansion methods have been used abundantly in the past to split the attached turbulent boundary layer into regions where dominant physical effects can be isolated and local solutions found. These methods naturally uncover the relevant scaling parameters of the flow and the functional nature of solutions. The works of Yajnik (1970) and Mellor (1972) have centered their arguments on the two-layered flow structure of Prandtl (1925) and Kármán (1930). The result is the establishment of a flow model where the sum of the viscous and turbulent stresses is considered to remain invariant across the entire near-wall region, the inner region (of thickness $\hat{\delta}=\operatorname{order}\left(v / u_{*}\right)$ ). In the adjacent outer layer (of thickness $\delta=\operatorname{order}\left(\left(u_{*} / u_{e}\right) L\right), L=$ external flow reference length, $u_{e}=$ external mean velocity), the solution is a small perturbation to the external potential flow.

Sychev and Sychev (1987) offer a different interpretation to the flow asymptotic structure. In their analysis, an additional intermediate layer is considered (of thickness $\tilde{\delta}=$ $\left.\operatorname{order}\left(\left(u_{*} / u_{e}\right)^{2} L\right)\right)$, where a balance of inertia forces, the pressure gradient and turbulent friction forces occurs. Thus, they argue that the inclusion of this new layer is essential to explain flow separation under an adverse pressure gradient. In fact, the two-layered structure $(\hat{\delta}, \delta)$ does not permit turbulent friction forces to act on the velocity defect region to a first-order approximation. Also, in the inner region, it does not permit the pressure gradient to act on the dominating friction forces. These two difficulties must then be remedied by the inclusion of a third layer $(\tilde{\delta})$.

In view of the above arguments, near-wall solutions for flows subject to pressure gradients can be obtained if a balance between friction forces and the pressure gradient is considered to exist in some region of the flow. This region corresponds to an intermediate limit process so defined as to match the outer limit of the inner solution with the inner limit of the intermediate solution of Sychev and Sychev's flow structure. Thus, we may write for this region

$$
\mu \partial_{z}^{2} u-\partial_{z}\left(\rho \overline{u^{\prime} w^{\prime}}\right)=\partial_{x} p
$$

The above equation is the basis of the following discussion. 


\subsection{Attached Flow Over a Smooth Wall}

In the sense of Kaplun (1967) (see also Lagerstrom and Casten 1972; Cruz and Silva Freire 1998) — and under some appropriate intermediate limit process-Eq. 1 contains the approximate equations for two distinct limiting conditions: flow very near to a wall and flow approaching the intermediate region (Sychev and Sychev 1987).

\subsubsection{Viscous Region}

Approaching the wall, the turbulent term in Eq. 1 tends to zero $\left(\partial_{z}\left(\rho \overline{u^{\prime} w^{\prime}}\right) \approx 0\right)$, the implication being that just the viscous and pressure terms become significant. Then, for flows under a zero-pressure gradient $\left(\partial_{x} p=0\right)$, a double integration of Eq. 1 yields the linear solution

$$
u^{+}=z^{+},
$$

with $u^{+}=u / u_{*}, z^{+}=z /\left(v / u_{*}\right)$.

The assumption implicit in the linear solution is the very existence of a near-wall viscous region. Surfaces with a well-defined viscous sublayer are termed smooth surfaces.

\subsubsection{Fully Turbulent Region}

Moving away from the wall, the viscous term becomes negligible $\left(\mu \partial_{z}^{2} u \approx 0\right)$ and the turbulent term dominates Eq. 1. Then, by summoning the mixing-length hypothesis, the solution for zero-pressure gradient flows can be obtained from a double integration as

$$
u^{+}=\varkappa^{-1} \ln z^{+}+A,
$$

where $\varkappa(=0.4)$ and $A(=5.0)$ are parameters that have to be found experimentally. In fact, the value of $A$ is fixed through the empirically verified hypothesis that the logarithmic and linear profiles intercept at $z^{+}=11$.

The immediate implication of the linear and logarithmic solutions is that, for flow over a smooth surface, the near-wall relevant scales for velocity and length are $u_{*}$ and $v / u_{*}$.

\subsection{Separated Flow Over a Smooth Wall}

At a separation point, where $u_{*}=0$ and $\partial_{x} p \neq 0$, the approximate solutions given by Eqs. 2 and 3 break down. Hence, more appropriate solutions need to be considered to incorporate the pressure effects and the condition $\tau_{w}=0$.

\subsubsection{Viscous Region (Goldstein's Solution)}

In the viscous sublayer, Goldstein (1948) showed the local solution to be

$$
u^{p}=(1 / 2)\left(z^{p}\right)^{2},
$$

where $u^{p}=u / u_{p v}, z^{p}=z /\left(v / u_{p v}\right), u_{p v}=\left((v / \rho) \partial_{x} p\right)^{1 / 3}$. The Goldstein's solution can be simply obtained by a double integration of Eq. 1 in the viscous sublayer $\left(\partial_{z}\left(\rho \overline{u^{\prime} w^{\prime}}\right) \approx 0\right)$ near a separation point $\left(\partial_{x} p \neq 0, \tau_{w}=0\right)$. 


\subsubsection{Fully Turbulent Region (Stratford's Solution)}

Stratford (1959) considered the limiting solution for distances away from the wall, approaching the intermediate region of Sychev and Sychev (1987). Again, neglecting the viscous term in Eq. 1 and using of the mixing length hypothesis, the no-slip boundary condition and the fact that at a separation point $\tau_{w}=0$ gives

$$
u^{p}=\left(2 \varkappa^{-1}\right)\left(z^{p}\right)^{1 / 2},
$$

with $u^{p}$ and $z^{p}$ defined according to Eq. 4 .

The implication is that the relevant velocity and length scales for flows at a separation point are $u_{p v}$ and $v / u_{p v}$.

Strictly speaking, the no-slip condition should not have been used by Stratford, for Goldstein's solution is the solution that is supposed to be valid at the wall. Equation 5 should then include an integration constant. Stratford also incorporated an empirical factor, $\beta=0.66$, to Eq. 5 to correct pressure rise effects on $\varkappa$.

\subsection{Extended Solution for Attached and Separated Flows Over a Smooth Wall \\ (Fully Turbulent Region)}

All approximate solutions derived so far have been obtained from local equations reduced from Eq. 1. Therefore, the global solution of Eq. 1 should also reduce, under the relevant limiting processes, to the local approximate solutions.

A double integration of Eq. 1 in the fully turbulent region $\left(\mu \partial_{z}^{2} u \approx 0\right)$ furnishes (see e.g., Cruz and Silva Freire 1998)

$$
u=2 \varkappa^{-1} \sqrt{\Delta_{w}}+\varkappa^{-1} u_{*} \ln \left(\left(\sqrt{\Delta_{w}}-u_{*}\right) /\left(\sqrt{\Delta_{w}}+u_{*}\right)\right)+C,
$$

with $\Delta_{w}=\rho^{-1} \tau_{w}+\left(\rho^{-1} \partial_{x} p\right) z$.

Equation 6 must be viewed with much discretion, for depending on the relative values of $\tau_{w}$ and $\left(\partial_{x} p\right) z$ the discriminant $\Delta_{w}$ might become negative, thus rendering the solution undetermined. Furthermore, the argument of the logarithmic term cannot become negative. In Cruz and Silva Freire (1998), three different cases have been identified and explicitly quoted.

In general, however, Eq. 6 can be seen as a generalization of the classical law of the wall for separating flows. In the limiting case $\left(\partial_{x} p\right) z \ll \tau_{w}$, Eq. 6 reduces to the logarithmic expression

$$
\begin{aligned}
& u^{+}=\varkappa^{-1} \ln z^{+}+b_{m}, \\
& b_{m}=2 \varkappa^{-1}+\varkappa^{-1} \ln \left(\left(u_{p \nu}^{3} / 4 u_{*}^{3}\right) e^{\varkappa C}\right) .
\end{aligned}
$$

Near a point of separation Stratford's solution is recovered.

In principle, Eq. 6 can be used indistinctly in all flow regions-including regions of reversed flow-provided the domain of validity of its discriminant is respected and appropriate integration constants are determined. Equation 6 cannot be written in terms of the similarity variables $u_{*}$ and $u_{p v}$ for in situations where any of these two parameters approaches zero, a singularity occurs. Thus, it will be kept in its present form.

Other different treatments of the lower boundary condition can be found in the literature to model separating flows. For two-dimensional, smooth, steep hills, Loureiro et al. (2007a) have investigated the performance of the formulations introduced by Mellor (1966), by Nakayama and Koyama (1984) and by Cruz and Silva Freire (1998). 


\subsection{Attached Flow Over a Rough Wall (Fully Turbulent Region)}

The effects of roughness on a boundary layer are dramatic. Provided the characteristic size of the roughness elements are large enough, a regime can be established where the flow is turbulent right down to the wall (fully rough flow). One important consequence is that the viscous sublayer is completely removed so that the linear and Goldstein's solutions do not apply anymore. The roughness also distorts the logarithmic profile acting as if the entire flow is displaced downwards.

The manner in which the logarithmic law is expressed to describe flow over a rough surface depends on the field of application. In meteorology, the common practice is to write

$$
u^{+}=x^{-1} \ln \left((z-d) / z_{0}\right),
$$

where $z$ is the distance above the actual ground surface.

The specification of the lower boundary condition on rough walls depends thus on two unknown parameters: the aerodynamic surface roughness, $z_{0}$, and the displacement height, $d$. Many works have attempted to relate the magnitude of $d$ and $z_{0}$ to geometric properties of the surface. Garratt (1992) mentions that the simple relation $d / h_{c}=2 / 3\left(h_{c}=\right.$ height of canopy) seems to offer good results for much of natural vegetation of interest. However, since $d$ is known to depend strongly on the way roughness elements are packed together, much discretion must be considered in using this relation. Garratt (1992) also mentions that many texts suggest considering $z_{0} / h_{c}=0.1$; typical natural surfaces satisfy $0.02<z_{0} / h_{c}<0.2$.

\subsection{Separated Flow Over a Rough Wall (Fully Turbulent Region)}

The arguments that lead to Stratford's law are based on the fundamental hypothesis that near a separation point a fully turbulent region can be identified in the flow. This consideration remains valid for flow over a rough surface. The direct implication is that the procedure that resulted in the derivation of Eq. 5 can be repeated for flow over rough surface but with $z^{p}=(z-d) / z_{0}$ and $u_{p v}=\left(\left(z_{0} / \rho\right) \partial_{x} p\right)^{1 / 2}$. The integration constant must also be determined so as to correctly account for the roughness effects.

\subsection{Extended Solution for Attached and Separated Flows Over a Rough Wall (Fully Turbulent Region)}

The derivation of Eq. 6 disregards any detail of the wall roughness. This equation is, in fact, supposed to be valid not in the region adjacent to the wall where the complicated flow around the individual roughness elements is apparent, but, instead, in a region where the flow statistics are spatially homogeneous. Hence, inasmuch as for the classical law of the wall, the characteristics of the rough surface must enter the problem through the integration constant $C$. In addition, the coordinate system must be displaced by $d$. The immediate conclusion is that Eq. 6 can be used to model separating flow over a rough surface provided $d$ and $C$ are adequately modelled.

Parameter $C$ is a general function of $\tau_{w}, \partial_{x} p$ and $z_{0}$ that must be determined by a consistent analysis of experimental data. However, an estimate of its functional form might be obtained by considering the limiting behaviour of Eq. 6 as $\tau_{w} \gg\left(\partial_{x} p\right) z$. The resulting expression is

$$
C=\varkappa^{-1} u_{*}\left[\ln \left(4 u_{*}^{2} /\left(\left(\rho^{-1} \partial_{x} p\right) z_{0}\right)\right)-2\right] .
$$

This parametrization scheme is presented here for the first time. 


\section{Experiments}

\subsection{Water Channel, Model Hill and Roughness}

The formulation presented in the previous section will be tested against data obtained in a water channel.

The general characteristics of the channel have been described in Loureiro et al. (2007a,b). The channel is housed in the Hydraulics Laboratory of the Civil Engineering Department of the University of Oporto. The total channel length is $17 \mathrm{~m}$ and the cross-sectional area is $0.40 \mathrm{~m}$ wide by $0.60 \mathrm{~m}$ high. The sides of the channel are made of glass and the bottom is made of acrylic.

The water circulation system provides a maximum volumetric flow rate of $1501 \mathrm{~s}^{-1}$. During a typical run, two pumps are used to maintain the maximum flow rate variation within \pm 0.8 $\%$. A set of screens and filters is used to stabilize the flow and suppress any excessive level of turbulence. The screens and filters also control the grain-size of particles in suspension. A magnetic flowmeter is used to keep the flow rate uncertainty within $0.0011 \mathrm{~s}^{-1}$, and the water level is controlled through a vertical steel gate. In the present measurements, two flow rates were used: 2.65 and $26.761 \mathrm{~s}^{-1}$.

The hill model was set $12 \mathrm{~m}$ downstream of the channel entrance; its shape follows the same Witch of Agnesi profile used by Loureiro et al. (2007a,b). The hill is thus defined by

$$
z_{H}=H_{1}\left[1+\left(x / L_{H}\right)^{2}\right]^{-1}-H_{2},
$$

where $H\left(=H_{1}-H_{2}\right)(=60 \mathrm{~mm})$ is the hill height and $L_{H}(=150 \mathrm{~mm})$ is the characteristic length representing the distance from the crest to the half-height point. The maximum hill slope is $18.6^{\circ}$. Coordinates $x$ and $z$ represent the longitudinal and vertical axes, respectively.

The roughness elements consisted of rigid rubber strips $3 \mathrm{~mm}$ wide by $3 \mathrm{~mm}$ high that were spaced $9 \mathrm{~mm}$ apart, and the rough surface extended from $1.5 \mathrm{~m}$ upstream of the hill top to $1.5 \mathrm{~m}$ downstream. The geometric details of the hill and of the roughness are given in Fig. 1.

\subsection{Instrumentation}

A two-component Dantec laser Doppler anemometry(LDA) system using an Ar-ion tube laser was operated in the forward scatter mode to measure mean and fluctuating velocity fields. A Bragg cell unit was used to introduce a digitally-controlled electronic shift that was necessary to resolve the direction of the flow field and give correct measurements of near-zero mean velocities. The light beams were made to pass through a series of conditioning optical elements to achieve a small measurement volume and to improve the optical alignment. Front lenses with $500 \mathrm{~mm}$ focus length were mounted on the probe to accurately position the measurement volume on the centreline of the channel. Before being collected by the photomultipliers, the scattered light was made to pass through interference filters of 514.5 and $488 \mathrm{~nm}$, so that only green and blue light were received on each photomultiplier, respectively. Table 1 lists the main characteristics of the laser Doppler system used. The signals from the photomultipliers were band-pass filtered and processed by a burst spectrum analyzer operating in a single measurement per burst mode. A series of LDA biases were avoided by adjusting the strictest parameters on the data processor. The level validation and the signal-to-noise ratio were 8 and 5 respectively. For simultaneous measurements of longitudinal and vertical velocities, a coincidence window of 5,000 $\mu$ s was used. For the statistics at each point, 20,000 samples were considered. 

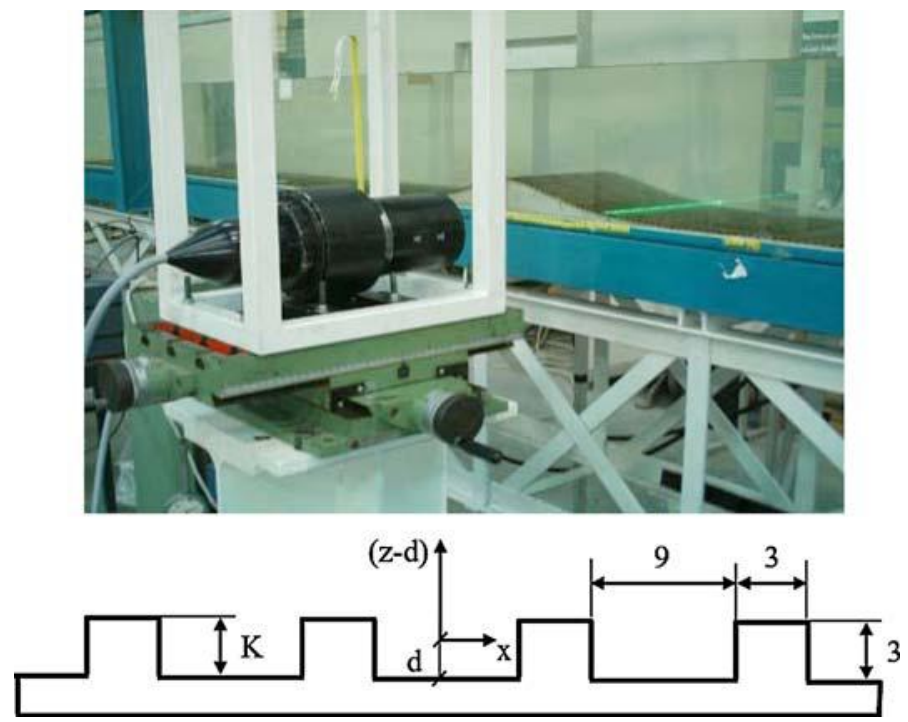

Fig. 1 Illustration of model hill and details of rough surface. Dimensions are in $\mathrm{mm}$. $K=$ height of the roughness elements, $d=$ displacement height, $z=$ distance from the bottom of the roughness elements

Table 1 Main characteristics of the laser Doppler system

\begin{tabular}{ll}
\hline Wavelength & $514.5 \mathrm{~nm}$ (green) $488 \mathrm{~nm}$ (blue) \\
Half-angle between beams & $1.604^{\circ}$ \\
Fringe spacing & $9.191 \mu \mathrm{m}$ (green) $8.718 \mu \mathrm{m}$ (blue) \\
Beam spacing & $28 \mathrm{~mm}$ \\
Beam diameter & $2.2 \mathrm{~mm}$ \\
Dimensions of the & \\
$\quad$ measurement volume & \\
Major axis & $5.31 \mathrm{~mm}$ (green) $5.04 \mathrm{~mm}$ (blue) \\
Minor axis & $149.0 \mu \mathrm{m}$ (green) $141.0 \mu \mathrm{m}$ (blue)
\end{tabular}

Typical uncertainties associated with the mean velocity data-U, $W$-are below $0.2 \%$ of the free stream velocity, $u_{\delta}$. In regions of reverse flow, the uncertainties increase to about $0.3 \%$ of the free stream velocity. Regarding the Reynolds stress components- $\overline{u^{\prime} u^{\prime}}, \overline{w^{\prime} w^{\prime}}, \overline{u^{\prime} w^{\prime}}-$ uncertainties were estimated to be $2.3,1.8$ and $4.2 \%$ of the square of the friction velocity of the undisturbed flow, respectively. In regions of reverse flow, 3.8, 3.5 and $6.9 \%$ are typical values.

\section{Results}

As mentioned above, formulation of the lower boundary condition given by Eq. 6 are tested against three different flow conditions: the smooth wall data of Loureiro et al. (2007b) SS - and two new datasets of flow over rough surface-RSA and RSB. Measurements were conducted on the channel centreplane. In all, 36 stations have been considered, and are shown in Fig. 2. 
Fig. 2 Location of measuring stations and coordinate system. (a) Loureiro et al. (2007b) (SS, $R_{\delta}=4,772$ ), (b) present (RSA, $\left.R_{\delta}=4,425\right)$, (c) present (RSB, $\left.R_{\delta}=31,023\right)$
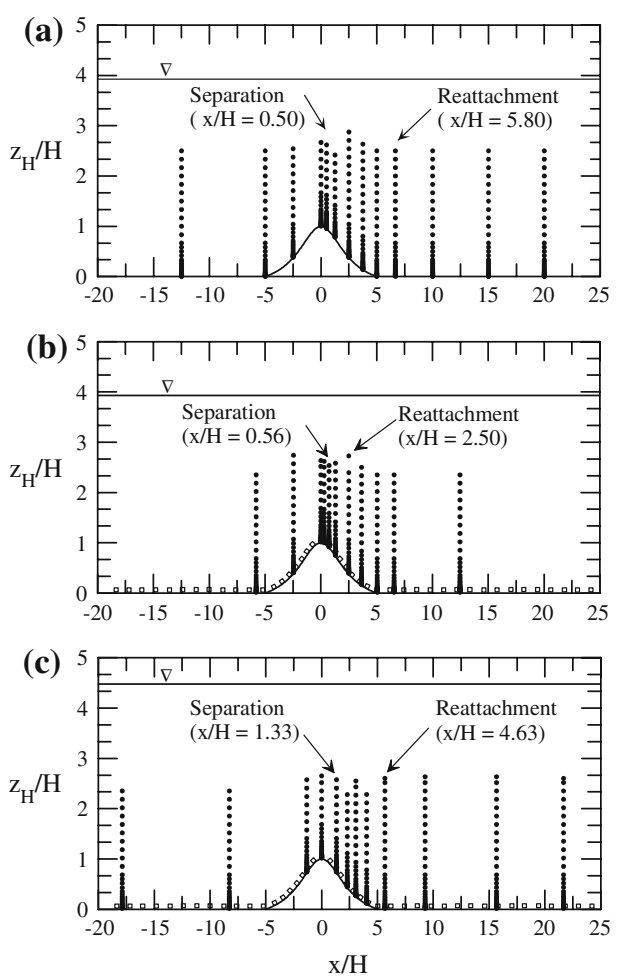

Table 2 Properties of undisturbed profile

\begin{tabular}{llll}
\hline Property & SS & RSA & RSB \\
\hline Boundary layer thickness $(\delta, \mathrm{mm})$ & 100 & 90 & 100 \\
External velocity $\left(u_{\delta}, \mathrm{m} \mathrm{s}^{-1}\right)$ & 0.0482 & 0.0497 & 0.3133 \\
Friction velocity $\left(u_{*}, \mathrm{~m} \mathrm{~s}^{-1}\right)($ Clauser $)$ & 0.0028 & 0.0047 & 0.0204 \\
Friction velocity $\left(u_{*}, \mathrm{~m} \mathrm{~s}^{-1}\right)\left(\overline{u^{\prime} w^{\prime}}\right)$ & 0.0023 & 0.0043 & 0.0225 \\
Displacement height $(d, \mathrm{~mm})$ & 0.0 & 2.1 & 2.0 \\
Roughness length $\left(z_{0}, \mathrm{~mm}\right)$ & 0.08 & 0.83 & 0.33 \\
Reynolds number $\left(R_{\delta}\right)$ & 4,772 & 4,425 & 31,023 \\
Reynolds number $\left(R_{z_{0}}\right)$ & 0.22 & 3.88 & 6.65 \\
\hline
\end{tabular}

\subsection{Undisturbed Boundary Layer}

In Loureiro et al. (2007b), it was shown that smooth-wall mean velocity profiles at stations upstream of $x / H=-12.5$ compare very well with the mean velocity profile in the absence of the hill at station $x / H=0$. We can also anticipate that, for the rough surface data (RSB, Table 2), measurements made at stations $x / H=-17.87$ and -8.27 do not differ appreciably between each other. On this evidence, the properties of the undisturbed boundary-layer profiles are given here in terms of reference profiles considered at large upstream distances from the coordinate system origin. The main flow parameters are shown in Table 2.

The data in Table 2 regarding $u_{*}, d$ and $z_{0}$ need explanation, as given in the following. However, before embarking on the specifics regarding the determination of the flow 

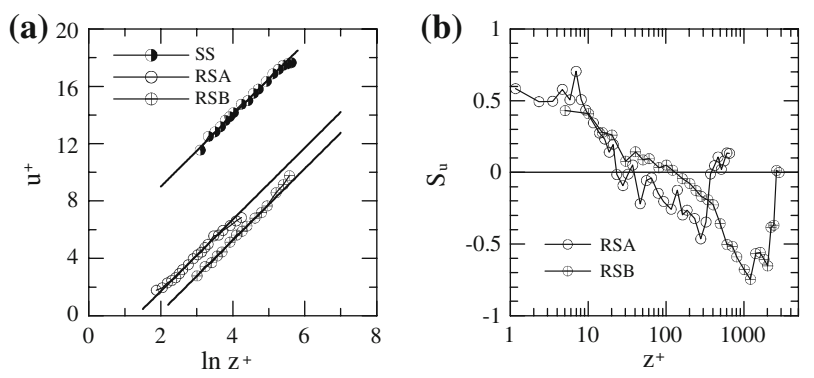

Fig. 3 Characterization of the flow regime. (a) Mean velocity profiles, (b) skewness profiles; $S_{u}=$ $\left.\overline{u^{\prime 3}} / \overline{\left(u^{\prime 2}\right.}\right)^{3 / 2}$

parameters, a brief discussion on whether conditions RSA and RSB yield aerodynamically rough flows is needed.

Schlichting (1979, p. 617) offers a physical interpretation of the flow regimes in the following terms: (i) a regime is said to be hydraulically smooth provided "the size of the roughness is so small that all protrusions are contained within the laminar sub-layer" $(0 \leq$ $k_{s}^{+} \leq 5, k_{s}^{+}=\left(k_{s} u_{*}\right) / v, k_{s}=$ sand grain roughness), (ii) in the transition regime, "protrusions extend partly outside the laminar sub-layer and the additional resistance, as compared with a smooth pipe, is mainly due to the form drag experienced by the protrusions in the boundary layer" $\left(5 \leq k_{s}^{+} \leq 70\right)$; (iii) in the fully rough regime, "all protrusions reach outside the laminar sub-layer and by far the largest part of the resistance to flow is due to the form drag which acts on them" $\left(k_{s}^{+} \geq 70\right)$.

The classification intervals defined above are mentioned by Schlichting (1979) to have been obtained by Nikuradse with maximum density sand. In practical applications, the typical roughness density is much smaller, so that roughness cannot be described by the indication of the height of a protrusion, $k$. Schlichting himself has proposed a scale of standard roughness and adopted Nikuradse's sand roughness for correlation (see e.g., his Fig. 20.24 and note the large differences between $k$ and $k_{s}$ ).

If Schlichting's advice is taken into consideration, the above intervals become $0 \leq z_{0}^{+} \leq$ $0.17,0.17 \leq z_{0}^{+} \leq 2.34$ and $z_{0}^{+} \geq 2.34$. In the present study, $z_{0}^{+}=3.88$ (RSA) and 6.65 (RSB). Thus, in principle, both RSA and RSB qualify as fully rough surfaces. In fact, Snyder and Castro (2002) have shown that for the type of rough wall used in laboratory simulations - arrays of sharp-edged obstacles with significant separation between each other-a fully rough regime can be obtained for a critical Reynolds number as low as unity $\left(R_{z_{0}}=1\right)$. The use of $z_{0}$ as the appropriate scale defining a roughness has been further emphasized by Castro (2007).

Figure 3 shows the undisturbed mean velocity and skewness profiles in inner variables for conditions SS, RSA and RSB. The fully rough portions of the boundary layers are identified in Fig. 3a. Some authors have preferred to characterize the effects of wall roughness in terms of the shift of the log-portion of the velocity profile, $\Delta u^{+}$; (for a discussion on $\Delta u^{+}$including its definition, see, e.g., Snyder and Castro 2002; Castro 2007). As expected, an increase in $R_{z_{0}}$ results in an increase in the roughness function, $\Delta u^{+}$. In most works, values of $\Delta u^{+}$ typically range between 5 and 10 . Here, we found $\Delta u^{+}=7.3$ (RSA) and 8.7 (RSB). Hence, Fig. 3a undoubtedly indicates that conditions RSA and RSB correspond to aerodynamically rough flows.

As quoted in Castro (2007), $z_{0}^{+}$and $\Delta u^{+}$are entirely equivalent measures of the roughness and are related via a simple algebraic expression. Arguments based on $z_{0}^{+}$and $\Delta u^{+}$are given here to favour readers inclined to any of the two possible formulations. 
Additional information on the structure of turbulent flows can be extracted from the higher order moments of the fluctuating velocities, skewness and flatness factors. In particular, for a smooth wall, the skewness factor of the longitudinal fluctuations $\left(S_{u}\right)$ increases monotonically as the wall is approached, reaching a peak value in the viscous sublayer that is followed by a region of rapid decrease. According to some authors (Andreopoulos et al. 1984; Fernholz et al. 1995) positive fluctuations of $u$ occur more frequently than large negative fluctuations in the viscous region as a result of the arrival of external high-speed fluid (sweep events) (Gad-el-Hak and Bandyopadhyay 1994). Other authors (Krogstad and Antonia 1999; Flack et al. 2007) report that $S_{u}$ remains negative across the entire boundary layer. For aerodynamically rough flows, $S_{u}$ is characteristically very high and positive $(\approx 0.5)$ in the near-wall region. The data in Fig. $3 \mathrm{~b}$ furnish therefore further evidence that conditions RSA and RSB correspond to aerodynamically rough flows.

Research on flow over rough surfaces has always suffered from the intrinsic difficulty of finding the wall shear stress. If surfaces have a well-defined geometry that allow pressure taps to be fitted to the roughness elements, the method of Perry et al. (1969) can be used. Otherwise, the two basic available indirect methods are: the graphical method of Clauser (1954) and the hypothesis of Prandtl (1925) that across the wall layer the total shear stress deviates just slightly from the wall shear stress.

The method of Clauser (1954) has been comprehensively reviewed by Perry and Joubert (1963). For flows over a smooth surface, two properties of a best fitted logarithmic line can be used to determine $u_{*}$, its slope and linear coefficient. On a rough surface, the logarithmic profile is deformed by the displacement height and shifted bodily by the roughness length. The implication is that the value of $u_{*}$ can only be confirmed by the slope of the logarithmic fit. This difficulty introduces much uncertainty into the estimation process (Perry and Joubert 1963).

The practice of using the average measured shear stress profile to find $u_{*}$ has been discussed by (Cheng and Castro 2002). The difficulties are also many. Clearly, the shear stress shows an appreciable longitudinal variation depending on its distance from the wall. In the roughness sublayer, a wavy variation in phase with the crest of the roughness elements can be observed. Farther away from the wall, in a region where the variations drop below $10 \%$, the inertial sublayer is defined (Oke 1987). The interesting fact reported by Cheng and Castro (2002) is that better logarithmic fits can be found for the mean velocity profile if spatially-averaged data in the roughness sublayer are used in preference to data in the inertial sublayer. The best fits, however, are observed to be obtained with neither of these values of $u_{*}$, but instead with values of $u_{*}$ evaluated directly from the form drag of the roughness elements. These latter values were found to be significantly higher than those obtained from the shear stress in either of the two regions considered (Cheng and Castro 2002).

A detailed description of the fitting procedure used in the present work can be found in Loureiro et al. (2007b). Here, only a brief explanation of aspects typically associated with roughness will be presented.

To find $u_{*}, d$ and $z_{0}$ the raw undisturbed velocity profiles were subtracted $0.1 \mathrm{~mm}$ from their distance to the wall. Then, a best fit logarithmic curve was searched in the near-wall region by inspection of the maximum coefficient of determination $\left(R_{s q}\right)$, R-squared (Loureiro et al. 2007b; Bevington 1969). The process was repeated—using the same subtraction—until the curve with the best R-squared coefficient could be identified. This curve, written in terms of the format defined by Eq. 8, then provides $u_{*}, d$ (Fig. 1) and $z_{0}$ directly for the undisturbed flow. Other statistical fitting parameters were also considered in this evaluation process. 
Fig. 4 Graphical method to find the displacement height. (a) Velocity profile reduction technique ( $z$ and $d$ are plotted in $\mathrm{m}$ ), (b) best fitting statistics

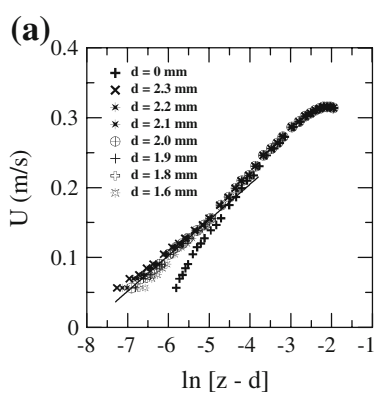

(b)

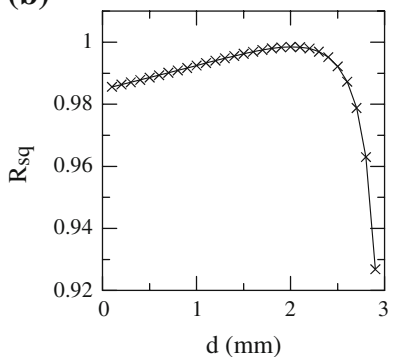

This graphical method described above is illustrated in Fig. 4. The velocity profile plotted in Fig. 4a corresponds to condition RSB, station $x / H=-17.87(x=-1072 \mathrm{~mm})$. The $R_{s q}$ values obtained for the various $d$ attempts are plotted in Fig. 4b. The existence of a welldefined maximum value is clear. A quick inspection of Fig. $4 \mathrm{~b}$ reveals that the appropriate value of $d$ is about $2 \mathrm{~mm}$, and the corresponding values of $R_{s q}$ are listed in Table 3 . Note that a final decision can only be made by inspection of the fourth decimal digit of $R_{s q}$. In fact, many authors have regarded the task of finding an accurate estimate of $d$ as one of extreme difficulty.

To evaluate $u_{*}$ from the Reynolds shear stress profile, a near-wall region with an approximate constant distribution of $-\overline{u^{\prime} w^{\prime}}$ was searched for. The friction velocity was then evaluated from $u_{*}=\sqrt{-\overline{u^{\prime} w^{\prime}}}$.

The mean velocity profiles for all undisturbed flow conditions are shown in Fig. 5. Curve fittings provided by Eqs. 6 and 9 are also presented, and the resulting local properties estimated from Eqs. 6 and 9 are shown in Table 4. These values can be compared with the values introduced in Table 2. In particular, the viscous region is clearly identified in Fig. 5a for the SS-condition. Thus, the local linear behaviour of the velocity profile can be used to find $u_{*}$. A rigorous estimate by Loureiro et al. (2007b) furnishes $u_{*}=0.00276(x / H=-12.5)$, which compares very well with 0.002849 given in Table 4 .

Table 3 Statistics for prediction of the displacement height

\begin{tabular}{ll}
\hline$d(\mathrm{~mm})$ & $R_{s q}$ \\
\hline 2.2 & 0.997932 \\
2.1 & 0.998391 \\
2.0 & 0.998485 \\
1.9 & 0.998319 \\
1.8 & 0.997967 \\
\hline
\end{tabular}

(a)

(b)

profiles for all three experimental conditions according to Eqs. 6 and 9 ( $z$ and $d$ are plotted in $\mathrm{m}$ ). (a) SS and RSA, (b) RSB
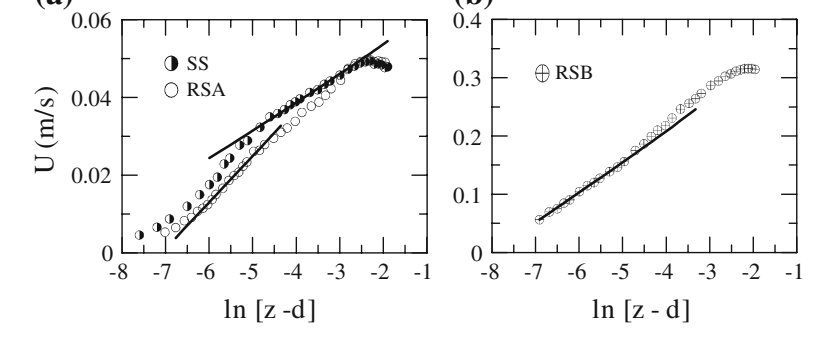
Table 4 Properties of undisturbed velocity profiles according to Eqs. 6 and 9

\begin{tabular}{lclll}
\hline Conditions & Station $(x / H)$ & $u_{*}\left(\mathrm{~m} \mathrm{~s}^{-1}\right)$ & $\rho^{-1} \partial_{x} p\left(\mathrm{~m} \mathrm{~s}^{-2}\right)$ & $C\left(\mathrm{~m} \mathrm{~s}^{-1}\right)$ \\
\hline SS & -12.50 & 0.002849 & 0.000015 & 0.058092 \\
RSA & -5.80 & 0.004736 & 0.000075 & 0.062406 \\
RSB & -17.87 & 0.020332 & 0.003347 & 0.269824 \\
RSB & -8.27 & 0.019224 & 0.009706 & 0.198584 \\
\hline
\end{tabular}

To search for values of $u_{*}$ and $\rho^{-1} \partial_{x} p$ in Eqs. 6 and 9 global optimization algorithms were used. In general, numerical algorithms for constrained nonlinear optimization can be categorized into gradient based methods and direct search methods. Gradient-based methods use first derivatives (gradients) or second derivatives (hessians), while direct search methods (nelder mead, differential evolution, simulated annealing, random search, etc.) do not use derivative information.

Direct search methods tend to converge more slowly, but can be more tolerant to the presence of noise in the function and constraints. Typically, algorithms only build up a local model of the problems. Also, many such algorithms insist on a certain decrease of the objective function, or decrease of a merit function-which is a combination of the objective and constraints - to ensure convergence of the iterative process. Such algorithms will, if convergent, only find local optima. For this reason they are called local optimization algorithms.

Global optimization algorithms, on the other hand, attempt to find the global optimum by allowing a decrease as well as an increase of the objective/merit function. Such algorithms are usually computationally more expensive. Here, four different methods were used for solution search: nelder mead, differential evolution, simulated annealing and random search. Only when all four methods furnished consistent results, with accuracy down to the sixth decimal fraction, was the search stopped.

\subsection{Flow Over the Hill}

The general flow patterns on the lee side of the hill for all three experimental conditions are shown in Fig. 6, where the extent of the regions of reverse flow is identified. The largest separated flow region is yielded by the smooth hill, $L_{S S}=x / H=5.30$ (Fig. 6a). The effects of wall roughness on the same flow conditions are shown in Fig. 6b. The region of reverse flow is substantially reduced in size $\left(L_{R S A}=x / H=1.94\right)$ and the separation point is slightly displaced downstream. An increase in Reynolds number by one order of magnitude causes the reverse flow region to enlarge again (Fig. 6c), but to a still smaller size $\left(L_{R S B}=x / H=3.30\right)$ than that exhibited in Fig. 6a for a smooth surface. The separation point is moved further downstream.

The estimated positions of the separation and re-attachment points for all three experimental conditions are seen in Fig. 2.

Velocity profiles on the upstream side of the hills and on their apexes are shown in Fig. 7. The fittings provided by Eqs. 6 and 9 are also shown. The flow properties are listed in Table 5 .

Any assessment of the predictions presented in Fig. 7 suffers, of course, from all the aforementioned difficulties regarding the determination of $u_{*}, \rho^{-1} \partial_{x} p, d$ and $z_{0}$. In addition, the identification of an appropriate coordinate system for flows over hills is a problem that poses many difficulties. Finnigan (1983) has suggested the use of physical streamlined coordinates, but unfortunately this is not an option for steep hills since large regions of reverse flow are formed. In Loureiro et al. (2007b), a detailed analysis of the uncertainties associated with the 
Fig. 6 General pattern of reverse flow region. (a) SS, (b) RSA, (c) RSB

Fig. 7 Velocity profiles on the upstream slope of the hills and on their apexes according to Eqs. 6 and 9 ( $z$ and $d$ are plotted in $\mathrm{m}$ ). (a) SS, (b) RSA, (c) RSB

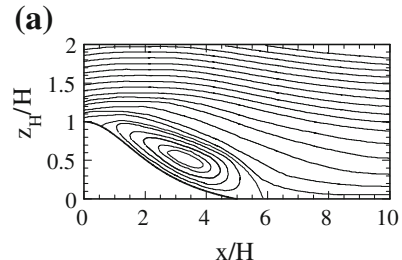

(b)

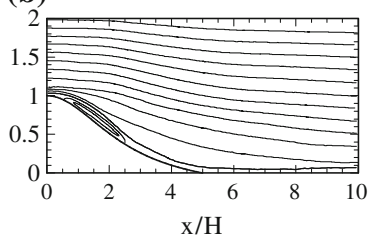

(c)

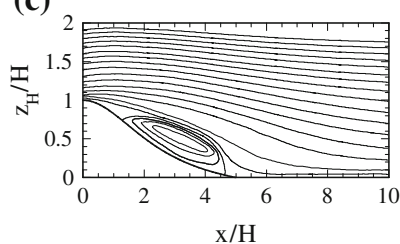

(a)

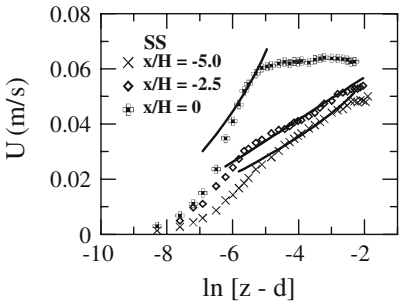

(b)

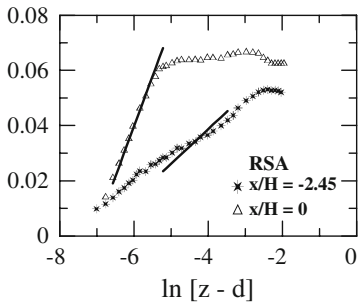

(c)

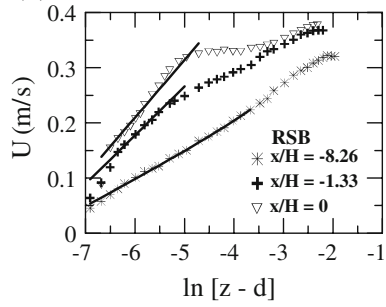

Table 5 Properties of the velocity profiles on the upstream side of the hills and on their apexes according to Eqs. 6 and 9

\begin{tabular}{lclll}
\hline Conditions & Station $(x / H)$ & $u_{*}\left(\mathrm{~m} \mathrm{~s}^{-1}\right)$ & $\rho^{-1} \partial_{x} p\left(\mathrm{~m} \mathrm{~s}^{-2}\right)$ & $C\left(\mathrm{~m} \mathrm{~s}^{-1}\right)$ \\
\hline SS & -5.00 & 0.002501 & 0.000174 & 0.034182 \\
SS & -2.50 & 0.003073 & $-2.9 \times 10^{-8}$ & 0.129097 \\
SS & 0.00 & 0.004246 & 0.013325 & 0.023270 \\
RSA & -2.45 & 0.005002 & $-2.8 \times 10^{-9}$ & 0.196491 \\
RSA & 0.00 & 0.014556 & $8.6 \times 10^{-7}$ & 0.449726 \\
RSB & -1.33 & 0.035262 & $1.3 \times 10^{-7}$ & 1.544860 \\
RSB & 0.00 & 0.041636 & $4.8 \times 10^{-8}$ & 2.018501 \\
\hline
\end{tabular}


Fig. 8 Velocity profiles near to a separation point. (a) RSA, (b) RSB

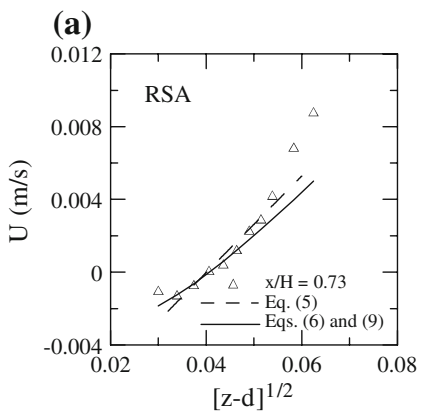

(b)

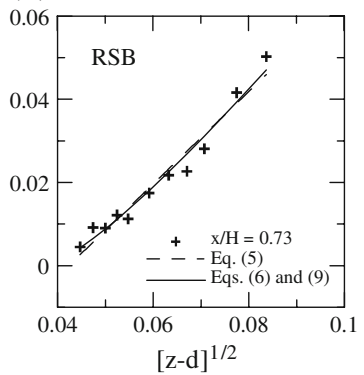

choice of a rectangular Cartesian system has been made. Typically, the uncertainties in the estimation of $u_{*}$ through logarithmic fits are $0.4 \%$ (flat wall) and $1.5 \%$ (curved wall). In the present work, the order of magnitude of these values should remain the same.

To find the best values of $u_{*}$ and $\rho^{-1} \partial_{x} p$, the same minimization procedure explained in the previous section was used, where the values of $d$ and $z_{0}$ were fixed from the undisturbed profiles. No alternative parametrization for $C$ in terms of $u_{*}, \rho^{-1} \partial_{x} p$ and $z_{0}$ was attempted in regions where the relation $\tau_{w} \gg\left(\partial_{x} p\right) z$ was not observed to hold; throughout this work, $C$ was defined by Eq. 9 .

The overall representation of the experimental data by Eqs. 6 and 9 is very good on the upwind side of the hill. For the SS-condition, well discriminated viscous and logarithmic regions can be identified, and consequently fits to the log-portion of the velocity profiles can be easily defined. For the rough wall, RSA and RSB-conditions, the complete absence of the viscous region prompted us to look for fits that extended right down to the wall. The procedure worked well except for profile $x / H=-1.33$, which exhibited a concave curvature that made it difficult to consider the three nearest points to the wall.

At the crest, predictions for the SS-condition were difficult to obtain through Eqs. 6 and 9. The strong flow acceleration enhanced the viscous effects, resulting in a linear velocity profile that spans at least the eight points nearest to the wall (Loureiro et al. 2007b). For the rough wall flows, however, very well-defined logarithmic profiles could be identified. The resulting log-fits furnished very small values for the pressure gradient, of the order of $10^{-6}$ or less, an expected result due to flow symmetry.

The behaviour of the velocity profiles near to a separation point is shown in Fig. 8 for the rough wall cases. A comparison between the experimental data and Eqs. 6 and 9, and Eq. 5 is also presented. Condition RSA does not result in a well-defined $z^{1 / 2}$-profile. In fact, the three points nearest to the wall are negative, a clear indication that the region of reverse flow has already been reached. Condition RSB, however, exhibits a reasonably well-defined Stratford type of solution at station $x / H=1.33$. Indeed, Eq. 5 shows a good agreement with the mean velocity data at $x / H=1.33$. Note that Eq. 5 implies explicitly that $u_{*}=0$. Equations 6 and 9 resulted in the following predictions: (i) very small and negative values of $u_{*}$, (ii) very large and positive values of $\rho^{-1} \partial_{x} p$. These results imply that station $x / H=1.33$ is located just past the separation point, in a region of reverse flow. Experimental evidence suggests that the flow position where $\tau_{w}$ is zero coincides with the position where instantaneous backflow occurs $50 \%$ of time $(\gamma=0.5)$. For flow over ribbed walls, the exact location of $\tau_{w}$ cannot be determined by direct measurement and the intermittency factor $\gamma$ has to be used. For this reason, finding experimentally the exact location of zero wall shear stress is a very 
Table 6 Properties of the velocity profiles at the separation points according to Eqs. 6 and 9, and Eq. 5

\begin{tabular}{lllclr}
\hline Conditions & $\begin{array}{l}\text { Station } \\
(x / H)\end{array}$ & Eq. & $u_{*}\left(\mathrm{~m} \mathrm{~s}^{-1}\right)$ & $\rho^{-1} \partial_{x} p\left(\mathrm{~m} \mathrm{~s}^{-2}\right)$ & $C\left(\mathrm{~m} \mathrm{~s}^{-1}\right)$ \\
\hline RSA & 0.73 & $(6)$ & -0.001140 & 0.003361 & -0.015761 \\
RSA & 0.73 & $(5)$ & 0.0 & 0.002819 & -0.010639 \\
RSB & 1.33 & $(6)$ & -0.008088 & 0.088580 & -0.096933 \\
RSB & 1.33 & $(5)$ & 0.0 & 0.049461 & -0.047103 \\
\hline
\end{tabular}

Fig. 9 Velocity profiles on the lee side of hills according to Eqs. 6 and 9 ( $z$ and $d$ are plotted in $\mathrm{m})$. (a) SS, (b) RSA, (c) RSB
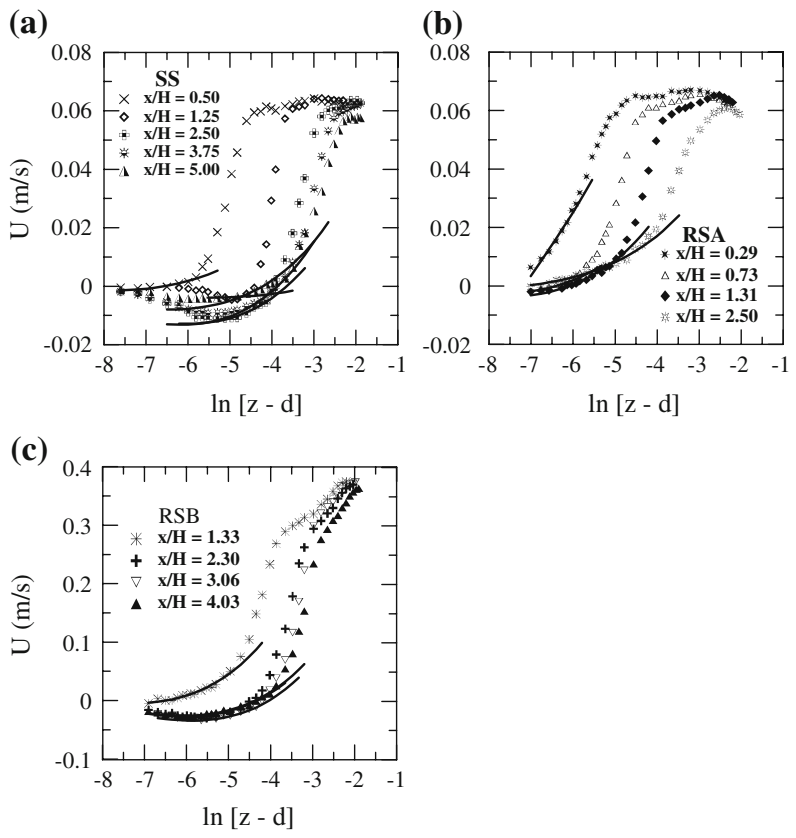

difficult task. In any event, station $x / H=1.33$ provides data that approximate very well the $z^{1 / 2}$-profile of Stratford.

At a separation point, and, for that matter, in regions of reverse flow, the present calculations have shown that a very small value of $z_{0}$ must be adopted. This is a very intuitive result since in regions of dead fluid the details of the surface should not matter at all in drag prediction. In the present work we have taken $z_{0}=O\left(10^{-6}\right) \mathrm{m}$.

The flow properties near to a separation point for conditions RSA and RSB are shown in Table 6. In reducing the data through Eq.5, the constant $\beta(=0.66)$ has not been considered.

The adequacy of Eqs. 6 and 9 in regions of reverse flow is shown in Fig. 9. For the SS-conditions, the low velocities in the vicinity of the wall result in a thick viscous-dominated sublayer, and the flow is then best represented by a Goldstein's solution (Loureiro et al. 2007b). A log-portion of the velocity profile can be identified, nevertheless, as shown in Fig. 9a. The resulting predictions of $u_{*}$ (Table 7) show a general good agreement as compared with the data of Loureiro et al. (2007b).

For the rough wall cases, the fits provided by Eqs. 6 and 9 are very good. The destruction of the viscous sublayer results in profiles that agree very well with the proposed expressions. 
Table 7 Properties of the velocity profiles on the lee side of hills according to Eqs. 6 and 9

\begin{tabular}{llllr}
\hline Conditions & Station $(x / H)$ & $u_{*}\left(\mathrm{~m} \mathrm{~s}^{-1}\right)$ & $\rho^{-1} \partial_{x} p\left(\mathrm{~m} \mathrm{~s}^{-2}\right)$ & $C\left(\mathrm{~m} \mathrm{~s}^{-1}\right)$ \\
\hline SS & 1.25 & -0.000471 & 0.000056 & -0.009265 \\
SS & 2.50 & -0.001820 & 0.001334 & -0.033696 \\
SS & 3.75 & -0.001982 & 0.001862 & -0.035886 \\
SS & 5.00 & -0.001324 & 0.001078 & -0.023114 \\
RSA & 0.29 & 0.007467 & 0.012388 & 0.020048 \\
RSA & 1.31 & -0.001325 & $-3.3 \times 10^{-7}$ & -0.018955 \\
RSA & 2.50 & -0.000556 & 0.001229 & -0.007083 \\
RSB & 2.30 & -0.007836 & 0.025849 & -0.116808 \\
RSB & 3.06 & -0.008588 & 0.027181 & -0.130875 \\
RSB & 4.03 & -0.007782 & 0.0262271 & -0.115451 \\
\hline
\end{tabular}

Fig. 10 Velocity profiles downstream of hills according to Eqs. 6 and 9 ( $z$ and $d$ are plotted in m). (a) SS, (b) RSA, (c) RSB (a)

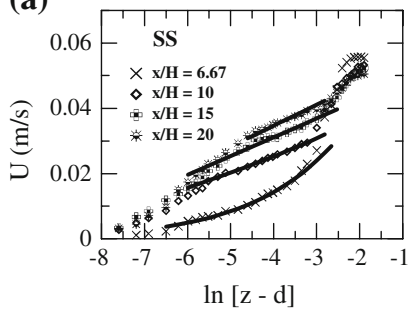

(b)

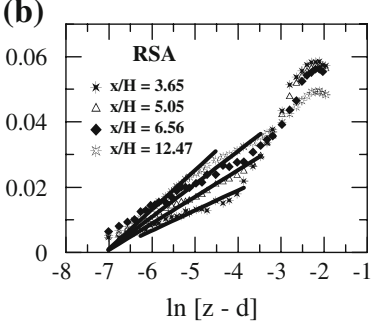

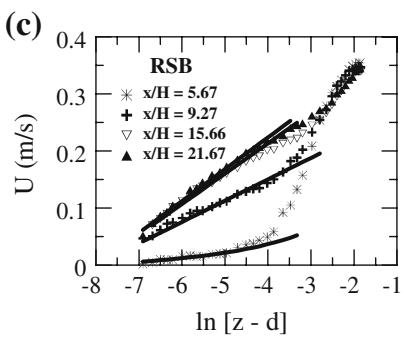

\subsection{Flow Downstream of the Hill}

The velocity profiles downstream of the hill are shown in Fig. 10. Close to the reattachment points ( $x / H=5.80$ (SS), 2.50 (RSA), 4.63 (RSB)) the velocity profiles should again approach a Stratford's solution. The predictions of Eqs. 6 and 9, however, furnish very good fits. At all other stations, log-regions can be identified. For the SS-condition, predictions of $u_{*}$ agree with the results of Loureiro et al. (2007b) to within $10 \%$. The fits to the rough wall cases are not good at $x / H=6.56$ (RSA). All other results seem consistent (Table 8).

\subsection{Skin Friction}

The friction-velocity consolidated results are shown in Fig. 11. In all flow regions the fits provided by Eqs. 6 and 9 resulted in consistent results. For the SS-condition, an independent assessment based on the results of Loureiro et al. (2007b) could be performed. For the rough surface cases, an assessment based on the classical law of the wall can be made for the far fields upstream and downstream of the hill. In regions close to separation or reattachment points (or regions of reverse flow), however, no standard method can be used to check $u_{*}$. 
Fig. 11 Friction-velocity according to Eqs. 6 and 9. (a) SS, (b) RSA, (c) RSB (a)

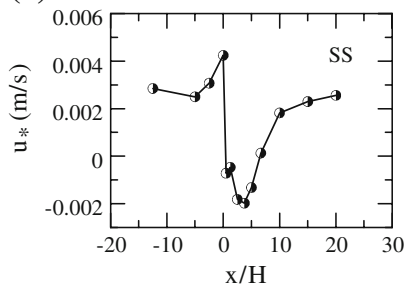

(c)

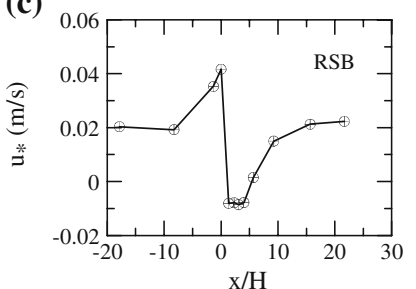

(b)

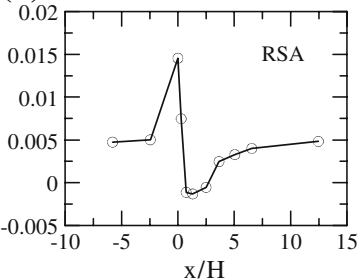

Table 8 Properties of the velocity profiles downstream of hills according to Eqs. 6 and 9

\begin{tabular}{lcccr}
\hline Conditions & Station $(x / H)$ & $u_{*}\left(\mathrm{~ms} \mathrm{~s}^{-1}\right)$ & $\rho^{-1} \partial_{x} p\left(\mathrm{~m} \mathrm{~s}^{-2}\right)$ & $C\left(\mathrm{~ms} \mathrm{~s}^{-1}\right)$ \\
\hline SS & 6.67 & 0.000129 & $4.78 \times 10^{-4}$ & -0.000473 \\
SS & 10.00 & 0.001822 & $5.13 \times 10^{-5}$ & 0.027606 \\
SS & 15.00 & 0.002298 & $1.15 \times 10^{-6}$ & 0.087586 \\
SS & 20.00 & 0.002561 & $4.20 \times 10^{-8}$ & 0.106459 \\
RSA & 3.65 & 0.002464 & $2.90 \times 10^{-8}$ & 0.076842 \\
RSA & 5.05 & 0.003279 & $-6.87 \times 10^{-6}$ & 0.104649 \\
RSA & 6.56 & 0.003997 & $1.06 \times 10^{-5}$ & 0.139854 \\
RSA & 12.47 & 0.004818 & $1.91 \times 10^{-8}$ & 0.162068 \\
RSB & 5.67 & 0.001461 & $2.99 \times 10^{-3}$ & 0.000560 \\
RSB & 9.27 & 0.014979 & $6.34 \times 10^{-8}$ & 0.692543 \\
RSB & 15.67 & 0.021243 & $4.10 \times 10^{-9}$ & 1.037510 \\
RSB & 21.67 & 0.022275 & $4.33 \times 10^{-9}$ & 1.129398 \\
\hline
\end{tabular}

In any event, the fact that Eqs. 6 and 9 have been derived from the first principles through asymptotic arguments and the mixing-length hypothesis- together with the very good fitting statistics—makes us believe that the present results are consistent.

\section{Final Remarks}

The present work has experimentally investigated the role of surface roughness on the behaviour of flow past a steep hill. Particular attention was dedicated to the study of the characteristics of the recirculation region. A neutrally stratified flow over a steep rough elevation was simulated in a water channel environment. Measurements of mean velocity were conducted with the aid of two-component laser Doppler anemometry. In all, 36 flow stations have been analyzed.

The study has striven to develop a near-wall solution capable of characterizing the flow in the following distinct regions: (i) the undisturbed region upstream of the hill, (ii) the upstream 
side of the hill, (iii) the hill apex, (iv) at a separation or reattachment point, (v) in regions of reverse flow, vi) the undisturbed region downstream of the hill. From what has been found, this can be achieved through Eqs. 6 and 9. In fact, the parametrization of $C$ is a subject that will deserve much consideration in the future. Here, a simple expression based on limit arguments has been proposed.

The present results allow a thorough description of the inner region of the boundary layer, providing good quality near-wall data to serve as a test case for numerical simulations.

Acknowledgements JBRL benefited from a Research Fellowship from the Brazilian Ministry of Science and Technology through Programme Prometro (Grant No 554391/2006-6). ASM benefited from a Research Scholarship from the Brazilian National Research Council (CNPq). ASM is also thankful to the Rio de Janeiro Research Foundation (FAPERJ) (Grant No E-26/171.198/2003) for the concession of further financial help regarding his stay at Oporto University. APSF is grateful to the Brazilian National Research Council (CNPq) for the award of a Research Fellowship (Grant No 306977/2006-0). The work was financially supported by CNPq through Grants No 477392/2006-7 and No 476091-2007/1, and by the Rio de Janeiro Research Foundation (FAPERJ) through Grants E-26/171.346/2005 and E-26/171.198/2003. ASM, JBRL and FTP are grateful to Prof. Maria Fernanda Proença of the Hydraulics Laboratory of Oporto University for all her help in setting up the flow rig, as well as for some very interesting technical discussions.

\section{References}

Allen B, Brown AR (2002) Large-eddy simulation of turbulent separated flow over rough hills. BoundaryLayer Meteorol 102:177-198

Andreopoulos J, Durst F, Zaric Z, Jovanovic J (1984) Influence of Reynolds number on characteristics of turbulent wall boundary layers. Exp Fluids 2:7-16

Bevington PR (1969) Data reduction and error analysis for the physical sciences. McGraw-Hill, New York, $351 \mathrm{pp}$

Brown AR, Hobson JM, Wood N (2001) Large-eddy simulation of neutral turbulent flow over rough sinusoidal ridges. Boundary-Layer Meteorol 98:411-441

Castro IP (2007) Rough-wall boundary layers: mean flow universality. J Fluid Mech 585:469-485

Castro IP, Apsley DD (1997) Flow and dispersion over topography: a comparison between numerical and laboratory data for two-dimensional flows. Atmos Environ 31:839-850

Cheng H, Castro IP (2002) Near-wall flow development after a step change in surface roughness. BoundaryLayer Meteorol 105:411-432

Clauser FH (1954) Turbulent boundary layers in adverse pressure gradients. J Aeronaut Sci 21:91-108

Cruz DOA, Silva Freire AP (1998) On single limits and the asymptotic behaviour of separating turbulent boundary layers. Int J Heat Mass Transfer 41:2097-2111

Cruz DOA, Silva Freire AP (2002) Note on a thermal law of the wall for separating and recirculating flows. Int J Heat Mass Transfer 45:1459-1465

Fernholz HH, Krause E, Nockemann M, Schober M (1995) Comparative measurements in the canonical boundary layer at $\operatorname{Re}_{\delta 2} \leq 6 \times 10^{4}$ on the wall of the DNW. Phys Fluids A 7:1275-1281

Finnigan JJ (1983) A streamlined coordinate system for distorted turbulent shear flows. J Fluid Mech 130:241258

Flack KA, Schultz MP, Connely JS (2007) Examination of a critical roughness height for outer layer. Phys Fluids 19:95-104

Gad-el-Hak M, Bandyopadhyay PR (1994) Reynolds number effects in wall-bounded turbulent flows. Appl Mech Review 47:307-365

Garratt JR (1992) The atmospheric boundary layer. Cambridge University Press, Cambridge, 316 pp

Goldstein S (1948) On laminar boundary layer flow near a position of separation. Quart J Mech Appl Maths 1: 43-69

Hewer FE (1998) Non-linear numerical model predictions of flow over an isolated hill of moderate slope. Boundary-Layer Meteorol 87:381-408

Hunt JCR, Leibovich S, Richards KJ (1988) Turbulent shear flow over low hills. Quart J Roy Meteorol Soc 114:1435-1470

Iizuka S, Kondo H (2004) Performance of various sub-grid scale models in large-eddy simulations of turbulent flow over complex. Atmos Environ 38:7083-7091 
Jackson PS, Hunt JCR (1975) Turbulent wind flow over a low hill. Quart J Roy Meteorol Soc 101:929-955

Kaplun S (1967) Fluid mechanics and singular perturbations. Academic Press, 369 pp

Kim HG, Patel VC (2000) Test of turbulence models for wind flow over terrain with separation and recirculation. Boundary-Layer Meteorol 94:5-21

Krogstad PA, Antonia RA (1999) Surface roughness effects in turbulent boundary layers. Exp Fluids 27:450460

Lagerstrom PA, Casten RG (1972) Basic concepts underlying singular perturbation techniques. SIAM Rev 14:63-120

Loureiro JBR, Soares DV, Fontoura Rodrigues JLA, Pinho FT, Silva Freire AP (2007a) Water tank and numerical model studies of flow over steep smooth two-dimensional hills. Boundary-Layer Meteorol 122:343-365

Loureiro JBR, Pinho FT, Silva Freire AP (2007b) Near wall characterization of the flow over a two-dimensional steep smooth hill. Exp Fluids 42:441-457

Mellor GL (1966) The effects of pressure gradients on turbulent flow near a smooth wall. J Fluid Mech 24:255-274

Mellor GL (1972) The large Reynolds number, asymptotic theory of turbulent boundary layers. Int J Eng Sci 10:851-873

Nakayama A, Koyama H (1984) A wall law for turbulent boundary layers in adverse pressure gradients. AIAA J 22:1386-1389

Oke TR (1987) Boundary layer climates. Routledge, London, 435 pp

Perry AE, Joubert PN (1963) Rough-wall boundary layers in adverse pressure gradients. J Fluid Mech 17:193211

Perry AE, Schofield WH, Joubert PN (1969) Rough-wall turbulent boundary layers. J Fluid Mech 37:383-413

Prandtl L (1925) Über die ausgebildete Turbulenz. ZAMM 5:136-139

Ross AN, Arnold S, Vosper SB, Mobbs SD, Nixon N, Robins AG (2004) A comparison of wind-tunnel experiments and numerical simulations of neutral and stratified flow over a hill. Boundary-Layer Meteorol 113:427-459

Schlichting H (1979) Boundary layer theory. McGraw Hill, New York, 817 pp

Snyder WH, Castro IP (2002) The critical Reynolds number for rough-wall boundary layers. J Wind Eng Ind Aerodyn 90:41-54

Stratford BS (1959) The prediction of separation of the turbulent boundary layer. J Fluid Mech 5:1-16

Sychev VV, Sychev VV (1987) On turbulent boundary layer structure. PMM USSR 51:462-467

Sykes RI (1980) An asymptotic theory of incompressible turbulent boundary-layer flow over a small hump. J Fluid Mech 101:647-670

von Kármán Th (1930) Mechanische ahnlichkeit und turbulenz. Proc Third Intern Congress for Appl Mech, Stockholm

Yajnik KS (1970) Asymptotic theory of turbulent shear flow. J Fluid Mech 42:411-427

Ying R, Canuto VM (1997) Numerical simulation of the flow over two-dimensional hills using a second-order turbulence closure model. Boundary-Layer Meteorol 85:447-474 